\title{
Lymphangioma of the tongue: case report
}

\author{
Ferrara F., Vessio G.*, Burgio A., Giannotti G., Bulotta A.L., \\ Pavone M., Meucci D., Messina M.
Division of Pediatric Surgery, Department of Pediatrics, Obstetrics and Reproductive Medicine, University Of Siena *Department of otolaryngology, University Of Siena

\begin{abstract}
Introduction. Lymphangiomas are uncommon congenital hamartomas of the lymphatic system, usually diagnosed in infancy and early childhood. Commonly located at head, neck extremities and genitals, they are rarely situated in the oral cavity. Preferred site of oral involvement is the tongue. The authors present a case of lymphangioma of the tongue treated with laser therapy.

Case report. The patient was a 7 years old female, that came to us for right upper quadrant abdominal pain. On examination we found the median lesion of the tongue in absence of symptoms. Thyroid scan was performed to exclude the presence of ectopic thyroid. Surgery was performed by excision of the lesion with CO2 $20 \mathrm{~W}$ by trans-oral laser therapy. The anatamopathological report posed diagnosis of lymphangioma. The follow-up to 8 months is in the norm: the aesthetic results are excellent and the patient doesn't report any symptoms.

Conclusion. This case had a very rare site of occurrence, the tongue, and was successfully managed with laser therapy. This surgical technique is very unusual among the various types of interventions but it allows good aesthetic results and good radical surgical excision, preserving vital structure.
\end{abstract}

Keywords: Lymphangioma of the tongue, laser terapy

\section{INTRODUCTION}

Lymphangioma is a localised malformation of the lymphatic system frequently located in the head and neck. Many of these lesions are congenital. They can be evident after surgery or trauma. Most of them are diagnosed before two years of age (1-2). The lymphangiomas represent about $6 \%$ of benign tumors of the smooth tissues (3).

It has been described for the first time by Redenbacher in 1828 , and it is classified as malformation and not as neoplasm (4).

Two theories have been advanced to describe the formation of the lymphatic system (5). The first is the centrifugal theory, whereby two endothelial buds grow from the jugular sacs and develop into lymph vessels. The second is the centripetal theory whereby an anastomotically developing lymphatic system eventually joins up to the central venous system.

Lymphangioma circumscriptum is a lymphatic malformation that is localised to the skin, subcutaneous tissue or sometimes muscle. The commonest sites are axillary folds, shoulders, flanks, proximal parts of the limbs and the perineum (6). There are few cases of lymphangioma circumscriptum of the oral cavity, but the most common site in this case is the tongue, especially the anterior part (7).

The authors present a case of lymphangioma of the tongue treated with laser terapy.

\section{CASE REPORTS}

The patient was a 7 years old female, who came to us for abdominal pain in right upper quadrant, because of gallstones.

On examination of the oral cavity, we found a median lesion of the tongue (Fig.1). The patient had no respiratory symptoms and no problems to swallow.

In suspicion of ectopic thyroid we did a scan. This examination excluded the possibility of ectopic thyroid. It was decided to perform surgical excision of the lesion. The excision was performed under general anesthesia with $20 \mathrm{~W} \mathrm{CO} 2$ by trans-oral laser therapy. The microscopic report made diagnosis of lymphangioma. At the follow-up at 8 months everything is normal: the aesthetic results are excellent and the patient does not report any symptoms.

\section{Correspondence to:}

Prof. Mario Messina, Division of Pediatric Surgery, Dept of Pediatrics, Obstetrics and Reproductive Medicine.

University of Siena. Policlinico “Le Scotte" - Viale Bracci - 53100 - Siena - Italy

Telefono: +39577586501 - Fax: +39577586174

E-mail: messinam@unisi.it 


\section{Discussion}

Lymphangiomas are uncommon, congenital tumours of the lymphatic system which mostly appears in children under five years as lobulated masses or cysts, usually in the head and neck. Involvement of the tongue, however, is rare. Lymphangioma of the tongue was first described by Virchow in 1854 (8). A lymphangioma is thought to be a contiguous mass of dilated lymphatics, and the cause is believed to be a developmental defect or primary malformation of the lymphatic channels. The management of a child with lingual lynphangioma involves various clinical features: respiratory obstruction, drooling for tongue protrusion, difficulty in chewing and swallowing and orthodontic abnormalities8. Sometime, the combined effects of these can make the child a social outcast requiring psychological support. Management is therefore complex and requires a team approach involving different specialities (9).

Lingual localisation presents specific therapeutic problems because of the microcystic character of the lesions and the marked functional problems (10). Various treatments have been attempted and proposed. Complete surgical excision is not possible because of the unacceptable degree of mutilation it would entail. Partial glossectomy, performed regularly, is a serious operation with a high rate of relapse or secondary growth and often results in a morphological modification of the tongue. Post-operative healing is painful and relatively long (2). The injection of sclerosing agents can be useful for macrocystic lesions but is not appropriate for microcystic lesions (11).

In our case the formation of the tongue was very small $(2 \mathrm{~cm})$. The patient did not show symptoms. For this reason, in our opinion, the best treatment was been the 20W CO2 Laser Therapy. This type of treatment allows a good control of bleeding, a good resection of lesion, with low the chances of recurrence, and preserves the structures of tongue, avoiding functional deficits.

\section{REFERENCES}

1. C. Gigue`re, N. Bauman, R. Smith. New treament options for lymphangioma in infants and children. Ann Otol Rhinol Laryngol 2002; 111: 1066-1075.

2. N. Leboulanger, G. Roger, A. Caze, O. Enjolras, F. Denoyelle, E.N. Garabedian. Utility of radiofrequency ablation for haemorrhagic lingual lymphangioma. Int Jou Pediat Otorhinolaryngology 2008; 72: 953-958.

3. J. N. Patel, J. Sciubba. Oral lesions in young children: Pediatric Clinics of North America. Pediatr Clin North Am 2003;: 50(2): 469-486.

4. C. M. Coffin, L. P. Dehner. Vascular tumours in childrenand adolescents: a clinicopathologic study of 228 tumoursin 222 patients. Pathol Annu, 1993; 28 (Pt 1): 97-120.

5. Th. Kennedy, M. Whitaker, P. Pellitteri, W.E. Wood. Cystic hygroma/lymphangioma: a rational approach to management. Laryngoscope 2001; 111 (11 Pt 1): 1929-1937.

6. P.S. Mortimer. Disorders of lymphatic vessels. In: Burns T, Breathnach S, Cox N, Griffiths C, eds. Rook's Textbook of Dermatology, 7th edn. London: Blackwell, 2004; 51.1-51.27. 7. A. Jamaroon, S. Pongsiriwet, S. Srisuwan, S. Krisanaprakornkit. Lymphangiomas of the tongue. Int J Pediatric Dentistry, 2003; 13: 62-63.

8. A. Balakrishnan, CM. Bailey. Lymphangiomaofthetongue, a review of pathogenesis, treatment and use of surface laser photocoagulation. J Laryngol Otol 1991; 105: 924-30.

9. D.S. Dinerman, E.N. Meyer. Lymphangioma macroglossia. Laringoscope 1976; 86: 291-296.

10. B. Cable, E.A. Mair. Radiofrequency ablation of lymphagio- matous macroglossia, Laryngoscope 2001; 111: 18591861 .

11. Okazaki, S. Iwatani, T. Yanai, H. Kobayashi, Y. Kato, T. Marusasa. Treatment of lymphangioma in children: our experience of 128 cases, J. Pediatr. Surg. 2007; 42(2): 386-389.
Fig. 1

Lesion of the tongue

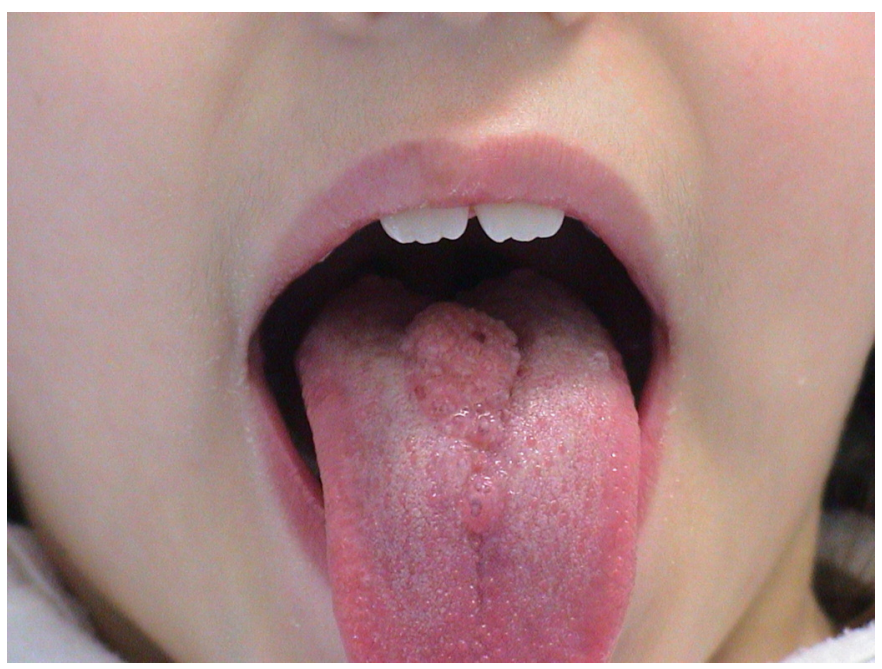

